\title{
Does the Fifth State of Matter Originate the Early
}

\section{Universe?}

\author{
Nagendra Nath Mondal \\ Department of Physics, Batanagar Institute of Engineering, Management and Science (Techno India Group), B-7/360 New word, \\ Putkhali, Maheshtala, Kolkata 700141, India
}

\begin{abstract}
In this article we have demonstrated BEC (Bose-Einstein Condensate) which is the fifth state of matter. Recent development of gamma-ray laser and scenario of black holes are highlighted. From the Stephen W. Hawking's lecture at KTH, Stockholm and the experimental evidence of LIGO scientific collaboration, USA, it is found that binary type black holes exist and can emit information which is detectable. Hence the source of information of black holes is not relay black; constituents are the densest state of material considered to be the fifth state and the primordial state of the Universe.
\end{abstract}

Key words: Black holes, BEC, cosmology, gamma-ray, laser, material, positronium.

\section{Introduction}

Twinkle twinkle little stars, how we wonder what you are! The stanza persuaded us to ask a common question, "What was the original state of this universe"? We have been trying to understand the nature of the primordial universe and find out the reasonable answers years after year. Four states of matter what we are observing in our daily lives: (1) Solid: particles in the material are closely packed and forces between particles are so strong that solid has a stable, definite shape and volume; (2) Liquid: it is an incompressible fluid that adopts the shape of a container because interatomic distance is larger than that of a solid, and molecules are mobile under the influence of external force; (3) Gas: it is a compressible state of matter where intermolecular forces are absent and intermolecular distance is larger than the molecular size. Hence, this state does not have any shape or volume; it covers all the space in the container; (4) Plasma: like gases plasma has no definite shape or volume. Unlike gases, plasmas are electrically conductive, produce magnetic field and

Corresponding author: Nagendra Nath Mondal, associate professor, research fields: astronomy \& astrophysics, high energy physics, material science and medical physics. current, and respond strongly to electromagnetic forces. Every star has this state from where enormous radiation with heat are emitting and spreading whole over the universe; (5) BEC (Bose-Einstein Condensate): it is a state of matter of dilute gas of bosons cooled to temperatures very close to absolute zero (order of nano Kelvin). Under such temperature, a large fraction of bosons occupy the lowest quantum state, at which point the macroscopic quantum phenomena become clear.

The latest model but not the least what is called Big Bang developed by Stephen Hawking almost 45 years back, a British theoretical physicist. He had revealed states of the early universe where first four states of matter are absent. We have been conceiving the veracity of this model rather than conflict. When he predicted that black holes-apparently inevitable gravitational pits-actually leak light, called $H R$ (Hawking radiation). "If you feel you are in a black hole, don't give up”, said Hawking. Anything that falls inside is thought to be ripped apart by the massive gravity, never to be seen or heard from again. It was an Information Paradox for physicists and they have tried to find a way for the information to escape the black hole's demise via the $H R$. Hawking unveiled 
a potential "answer" to the information-loss paradox-a way to give black holes hair-during a presentation given at the KTH Royal Institute of Technology in Stockholm on August 25, 2015: “I propose that the information is stored not in the interior of the black hole as one might expect but on its boundary, the event horizon," he said. [1]. In a paper published in January 2016 Hawking gives an explicit description of soft hair in terms of soft gravitons or photons on the black hole horizon, and shows that complete information about their quantum state is stored on a holographic plate at the future boundary of the horizon [2]. Recently researchers at the University of Kansas working with an international team at the LHC (Large Hadron Collider) in CERN have produced quark-gluon plasma-a state of matter thought to have existed right at the birth of the universe [3]. The material was discovered by colliding protons with lead nuclei at high energy inside the supercollider's Compact Muon Solenoid detector. Physicists have dubbed the resulting plasma the "littlest liquid". They described quark-gluon plasma as a very hot and dense state of matter of unbound quarks and gluons - that is, not contained within individual nucleons. It's believed to correspond to the state of the universe shortly about a microsecond after the Big Bang consisted of quark-gluon plasma.

Hence the state of our material world can be described with four known states: Solid, Liquid, Gas and Plasma. Plasma is the fourth state of mater where materials are neither liquid nor gas. Every star has this state from where enormous radiations with heat are emitting and spreading whole over the universe.

\section{Findings the Fifth State of Matter}

Very recently scientists have found the fifth state of mater experimentally which is called $B E C$ state. Theoretical formulation of this state was developed by Satyendra Nath Bose, a Bengali scientist and manipulated by Alfred Einstein, a German physicist that paves the way of forming $B E C$ in 1924 [4]. The critical temperature under which the $B E C$ occurred is given by $T_{c}=3.3125 \frac{h^{2} n^{2 / 3}}{4 \pi^{2} m K_{B}}$, where $n$ is the number of particle, $m$ is the mass of the particle, $K_{B}$ is the Boltzmann constant, and phase space density, $\rho=$ $n \lambda_{D}{ }^{3}$, where $\lambda_{D}$ is the de Broglie wavelength. In this state of matter atoms can't have different quantum states, at lowest possible temperature all those atoms condense at a single state is called Ground state by losing their kinetic energies. In $B E C$ atoms are confined in a tiny area that results enormous density ( $10^{18}$ atoms $/ \mathrm{cm}^{3}$ at nano Kelvin temperature). In 1995 scientists of BELL, Stanford University, NIST and MIT laboratories experimentally found $B E C$ of $H$, $\mathrm{He}, \mathrm{Ne}, \mathrm{Na}, \mathrm{Ru}$ atoms those lifetimes are infinite by utilizing advanced cooling technology. This remarkable achievement brings the Nobel Prize to Steven Chu, Claude Cohen Tannoudji, William D. Phillips and Wolfgang Ketterle after their successful experiments of BEC [5-10]. In 1982 Chu et al. determined the $1 S-2 S$ transition energy of Positronium (PS), an exotic atom of particle-antiparticle system using the laser cooling method and that paves him the way of Nobel Prize [11]. It has been known for many years that an electron $\left(e^{-}\right)$and its antiparticle, the positron $\left(e^{+}\right)$, may together form a metastable state, known as $P s\left(e^{+} e^{-}\right)$ atom. It is one of a family of leptonic hydrogen-like systems, includes muonium $\left(\mu^{+} e^{-}\right)$, tauonium $\left(\tau^{+} e^{-}\right)$as well as one of a family of more exotic atoms muium $\left(\mu^{+} \mu^{-}\right)$and protonium which are ideal test of QED (quantum electrodynamics), a theory of bound systems. Spin is a fundamental and intrinsic property of particle/antiparticle, and refers to their angular momentum. Spin polarized atoms are atoms that are all in the same spin state. A collection of spin polarized $P S$ atoms is needed to create $B E C$. According to the spin-spin interactions, two types of $P s$ with different lifetimes can be formed on the material surface after bombardment of slow positron 
beam: ortho $P S(o-P S)$, a spin triplet states whose life time is $142 \mathrm{~ns}$ and decay modes are odd multiple of $\gamma$-rays $(3 \gamma, 5 \gamma, 7 \gamma, \ldots)$ and para-Ps $(p-P s)$, a spin singlet state which has a lifetime of 125 ps and decay modes are even multiple of $\gamma$-rays $(2 \gamma, 4 \gamma, 6 \gamma, \ldots \ldots)$. Our laboratory at TMU (Tokyo Metropolitan University), Tokyo, Japan is famous for rare decay experiment. Para-Ps could not be the subject of a laser cooling experiment due to its shorter lifetime than the $1 S-2 P$ transition times of $o-P s$. Possibility of the $o-P s$ laser cooling is theoretically studied by Liang et al. utilizing the $1 S-2 P$ transition whose energy interval is $5.1 \mathrm{eV}$ corresponding to the transition photon wavelength of $243 \mathrm{~nm}$ [12]. It is a unique, well-defined exotic atom and hence the $B E C$ of $P S$ elucidates fundamental aspects of atomic and molecular physics as well as nano-technology. In spite of having a shorter lifetime (142 ns) than ordinary atoms produced at room temperature, it can be cooled down to a recoil limit of $0.1 \mathrm{~K}$ by ultra violet laser. During my stay at TMU we have studied this fact intensively [13-15].

\section{Results and Discussion}

In order to perform this challenging experiment and observe the $P S-B E C$ we have been developed a slow $e^{+}$beam line (TOPS) based on positron emitting radio isotope ${ }^{22} N a$, TOF (time-of-flight) spectrometer, a laser cooling system using a Cr:LiSAF crystal that creates a laser light with the wavelength of $972 \mathrm{~nm}$; and then using a second harmonic generator and a forth harmonic generator we achieved the required wavelength $243 \mathrm{~nm}$ for $o-P s$ excitation. Our laser cooling of $o-P S$ was the first proof-of-principle experiment towards realization of $P S-B E C$ at TMU in 2001 [15]. It was the most challenging experimental evidence for observing the fifth state of exotic atom. Bose-Einstein Condensation is increasingly of interest in many other branches of science. In Fig. 1a, theoretical distribution of $o-P s$ at laser on and laser off conditions is depicted and Fig. 1b experimental observation of lifetime distributions of $o-P s$ at laser on conditions with different temperatures is shown.

In 1946, Wheeler speculated that two $P s$ atoms may combine to form the di-Ps molecule $\left(P_{s_{2}}\right)$, with a binding energy of $0.4 \mathrm{eV}$. The researchers have purified the $P s$ sample effectively, which could lead to the development of a $\gamma$-ray laser [17]. It also could help scientists to understand why the universe is made up of matter and antimatter or just pure energy as stated in Big Bang cosmology. In 1994, two physicists Philip Platzman and Allen P. Mills Jr. at Bell Laboratory proposed that a $\gamma$-ray laser could be made from a $P s-B E C$. Following the brilliant success of our experiment in TMU, A. P. Mills \& D. Cassidy at the UCR (University of California, Riverside) created molecular $P s$, a brand-new substance, in the laboratory in 2007. Their experimental results at laser on/off conditions are shown in Fig. 1c. It is difficult to obtain low-energy positrons in large numbers; hence $P_{S_{2}}$ has not previously been observed [16, 17]. In order to make more intense slow positron beam, $e^{+}$ trapping systems Khaneko trap after the name of $\mathrm{J}$. Khaneko, a graduate student of our laboratory in TMU [13] and $e^{+}$bottle commonly known as Surko trap after the name of Clifford M. Surko at the UCSD (University of California, San Diego), USA [18], were developed respectively. Intense $e^{+}$bursts are implanted into a thin film of porous silica, $P s_{2}$ is created on the internal pore surfaces at the UCR. They found that molecule formation occurs much more efficiently than the competing process of spin exchange quenching, which appears to be suppressed in the confined pore geometry. This experimental result confirms the existence of the $P_{S_{2}}$ molecule and paves the way for further multi-Ps work. Using similar techniques, but with a more intense $e^{+}$source, they expect to increase the $P s$ density to the point where many thousands of atoms interact and can undergo a phase transition to form $B E C$. At such a high density, $P S$ atoms get annihilated simply by interacting with each other. But it turns out that not all the $P s$ atoms 
get annihilated under these conditions. In their Physical Review paper Cassidy explained that Ps atoms come in two types: an up type and a down type. The $P S$ atoms are only annihilated when an up type meets a down type. Two atoms of the same type do not affect each other. So if you have 50 percent ups and 50 percent downs and you squeeze them all together they will totally annihilate and turn into $\gamma$-rays. But if you have, for example, about 66 percent ups and 33 percent downs, then only half of the ups will be annihilated. You will get a load of $\gamma$-rays, but in the end you will be left with only one type of atom. In this case, up atoms. This is an important development for making the $B E C$, Cassidy said, because you have effectively purified your sample of $P s$. And you need a pure collection of spin aligned atoms to make the $B E C$. Non- $B E C$ atoms on the other hand whiz around at very high speeds, making them harder to study. Mills said that there are fundamental processes that can be looked at in new ways when you have matter in the $B E C$ state. Having $B E C$ atoms it is easier to probe the way they interact under certain conditions and make a $\gamma$-ray laser, which could have military and numerous scientific applications. A huge density of $\gamma$-ray laser of wavelength $511 \mathrm{keV}$ can be produced from the $P s-B E C$ as demonstrated in Fig. $1 \mathrm{~d}$. According to Mills and Cassidy, the new research could lead also to the production of fusion power, which is generated by nuclear fusion reactions. It could also one day help us to measure the gravitational interaction of antimatter with matter. At present, nobody knows for sure if antimatter falls up or down [19].

Wang et al. studied the $P s$-BECs applying the spinor matrix theoretically and found the difference of annihilation lifetimes between $o-P S$ and $p-P s$ by three orders of magnitude. In their paper, published in Physical Review A in 2014 they studied the spinor dynamics and annihilation processes in the $p-P s / o-P s$ system using both solutions of the time-dependent Gross-Pitaevskii equations and a semiclassical rate-equation approach [20]. The spinor interactions have an $O(4)$ symmetry which is broken to $S O(3)$ by an internal energy difference between $o-P S$ and $p-P s$. For an initially unpolarized condensate, there is a threshold density of $\sim 10^{19} \mathrm{~cm}^{-3}$ at which spin mixing between $o-P s$ and $p-P s$ occurs. Beyond this threshold, there are unstable spatial modes accompanied by spin mixing. To ensure a high production yield above the critical density, a careful choice of external field must be made to avoid the spin mixing instability.

A theoretical study suggested by Hamlet Avetissian at Yerevan State University that a new way to build a $\gamma$-ray laser from a $B E C$ of $e^{+} e^{-}$pairs. Gamma-ray lasers could have a number of important applications, from high-resolution imaging to new ways to probe or control nuclear transitions.

As reported in Physical Review Letters, Hamlet et al. [21] suggested that such a laser could be easier to build than previously thought, since it could be based on a single-pass scheme that does not rely on $\gamma$-ray mirrors. Although physicists have yet to make the highest density $P S-B E C$, recent advances in the manipulation of the short-lived particles suggest the authors' theory might soon be tested. Previous theoretical papers have investigated classical laser schemes, with the BEC gain medium placed between two mirrors. But the new work of Avetissian and colleagues shows that a simpler scheme could be possible: under certain parameter combinations, a very efficient cascade of annihilations, triggered by spontaneous emission, could determine lasing in one pass.

Researchers have developed a compact, circularly polarized $X$-ray source for studying biological materials. Many biological molecules absorb rightand left-handed circularly polarized light differently depending on their structure, so polarized light sources can be used to probe the shapes of molecules. In particular, coherent "soft" $X$-rays, which have wavelengths of up to a few tens of nanometers, are useful for imaging live cellular material. 


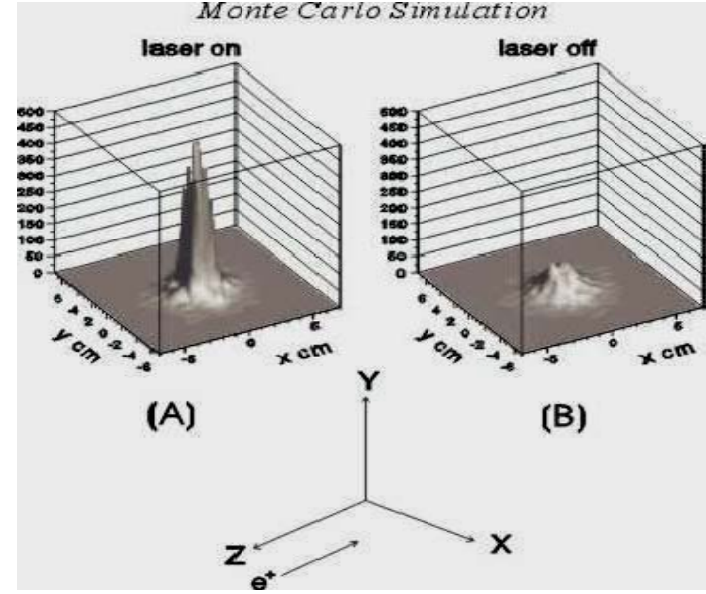

(a)

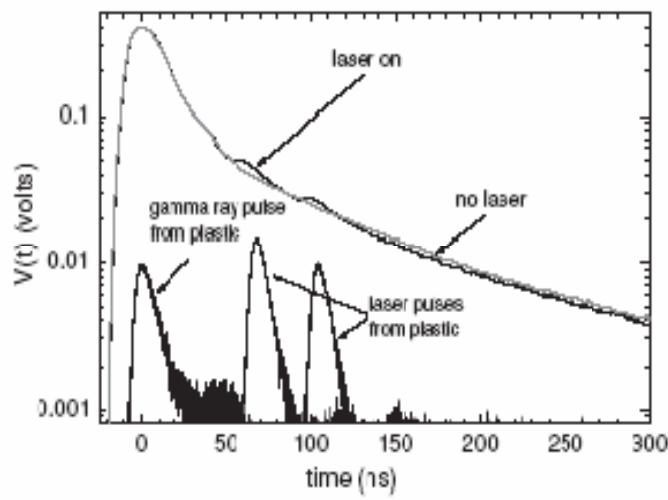

(c)

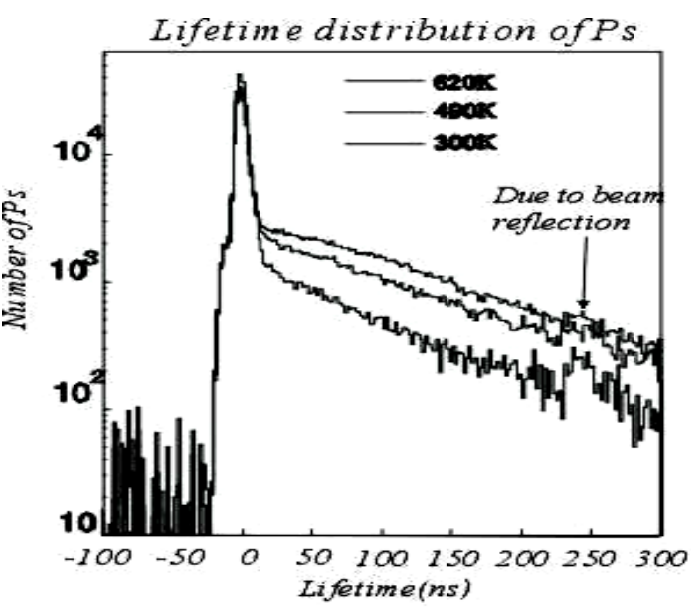

(b)

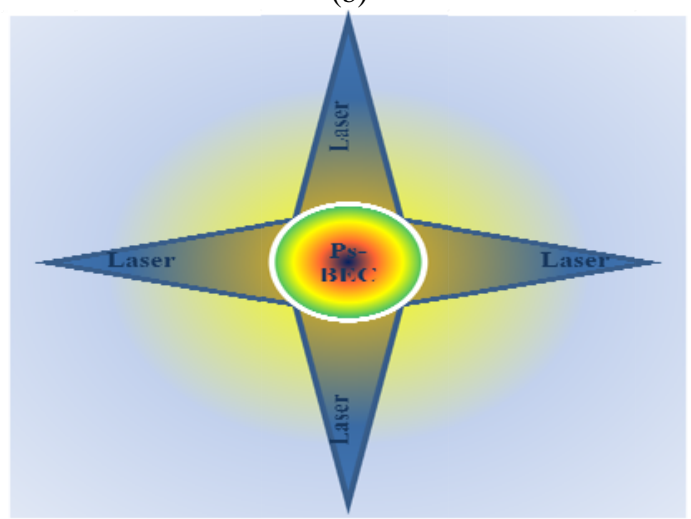

(d)

Fig. 1 (a) Theoretical attainment of laser cooling of o-Ps @ TMU; (b) Lifetime distribution of laser cooled o-Ps at different temperature@ TMU; (c) Laser cooled o-Ps @ UCR; (d) Production of coherence laser yet to be done from the Ps-BEC.

However, the majority of sources that can create such $X$-rays with high photon yields, for example, synchrotrons, are found at large-scale facilities, which are both expensive and extremely competitive to access. Now, researchers have developed and tested a compact, circularly polarized soft $\gamma$-ray laser that yields a large number of photons, yet is small enough to be used in a laboratory setting. A team led by Stéphane Sebban at Paris-Saclay University, France, has used a circularly polarized beam to seed lasing in plasma of krypton atoms and creates coherent light with a wavelength of 32.8 nanometers. The researchers have demonstrated that this approach amplifies the number of photons in the seed beam by nearly a factor of 10,000 , delivering more than 10 billion photons while keeping the radiation almost fully circularly polarized. The laser's pulses can last a few picoseconds and each contains approximately ten billion photons. This high photon yield is suitable for photon-demanding applications such as imaging proteins and viruses. Sebban and his colleagues suggest that their compact architecture can furthermore be scaled to shorter wavelengths and shorter pulse durations. These enhancements would open up new applications such as imaging nanostructures and studying demagnetization in thin films used in the electronics industry. More over this study will enhance the experiment of achieving the highest density $P s-B E C$ soon.

Trapping atoms near a photonic crystal waveguide produces strong atom-photon coupling that result in enhanced atomic emission of light. At the frontier between atomic physics and nano-photonics, researchers recently managed to trap single atoms 
around periodic structures called PCWs (photonic crystal waveguides). A new experiment advances this development by trapping multiple atoms near a PCW and inducing coherent emission (superradiance) from them. The results imply a strong atom-photon coupling that could be used to create quantum networks or explore many-body systems. A photonic crystal is a dielectric material containing nanoscopic elements in a periodic arrangement. Certain wavelengths of light propagate through the crystalline material what is called channeling had been initiated in 2000 by Prof. R. Hamatsu, my co-supervisor of doctoral thesis at TMU, while other wavelengths do not. By carefully designing the periodicity, scientists have exhibited surprising control of light in photonic crystals acting as low-loss waveguides. Recent work has also confined single atoms in close proximity to these PCWs, allowing novel interactions between guided photons and trapped atoms. Jeff Kimble and his colleagues at the CIT (California Institute of Technology) in Pasadena have designed a photonic system that traps multiple atoms (as many as three) and monitors their light output. Their waveguide is a flat, 50-micrometer-long slab of silicon nitride, etched into an alligator shape with corrugated edges and a thin gap down its spine. The researchers shined laser light from above, forming an atomic trap approximately 200 nanometers above the central gap. The team loaded this trap with cold cesium atoms and then injected pulses of light into the PCW at the alligator's head. The trapped atoms absorbed photons from the waveguide and then preferentially re-emitted photons back into the waveguide. The emission rate was proportional to the number of atoms in the trap - a signature of super radiance. The authors foresee tuning the strong atom-photon coupling to produce long-distance atomic interactions that could, for example, connect a network of atomic qubits. Scientists from the University of Maryland Joint Quantum Institute have calculated that it might be possible to build $\gamma$-ray lasers from mixing antimatter and matter together to make a compound $P s$, which would be used to turn ordinary light into a laser beam. They are trying to observe $P S-B E C$ with high density $P s$ production following the path shown by Cassidy et al. at UCR.

We have noticed from the above discussion that the highest intense $P s-B E C$ is yet to be attained for which many scientists have been developing advance science and technology. The problems which we should overcome first: (1 development of a higher density positron source $\left(>10^{12} \mathrm{e}^{+} / \mathrm{sec}\right)$, that is not yet done. Recently we are able to achieve $10^{9} e^{+} / \mathrm{sec}$ from the LINAC based $e^{+}$source at KEK, Japan. Scientists of JLC project (Prof. (Dr.) J. Urakawa @ KEK) and Spring-8 at Osaka, Japan, have been trying to develop this highest intense $e^{+}$beam line; (2) Development of a high density porous material target, the porosity is more than thousand billion per cubic $\mathrm{cm}$ where huge density of $e^{+}$can be trapped after thermalization in such a tiny area. The $e^{+}$annihilation laboratory, EPOS at Halle, Germany, where Prof. Reinhard Krause-Rehberg has been studying intensively in order to develop such a porous CPG glass target material that can trap huge density of $P s$ atoms; (3) Spectroscopy of $P s-B E C$. It is one of the most challenging scrap of $P s-B E C$ imaging. Here we need advance detection system that is capable to detect $\sim 100 \% e^{+} e^{-}$annihilation $\gamma$-rays energy and their time information. Image reconstruction algorithm must be written with the help of energy and time information and the position of each pixel of the position-sensitive detectors. Prof. R. Hamatsu and myself work together hard for the development of this type of spectrometer by utilizing the projects funded by the JSPS and the Ministry of Education, Culture, Sports, Science and Technology of Japan; (4) International collaboration: The last one decade progress on $P S-B E C$ shows that it is difficult for a single group of scientists from a particular laboratory in the world to attain the $P S-B E C$. Hence scientists of different expertise should gather on a single platform and work hand-to-hand for this 
wonderful achievement.

The recent progress on $P s-B E C$ and $\gamma$-ray laser persist most physicists to say that it is too early to know whether Hawking's idea is a real step forward. If he has solved the paradox successfully, to me it will provide another ingredient that is coming in, "what is the state of matter inside the black hole from where information is coming out”? To answer the larger mysterious question physicists will probably need not just a better understanding of black holes but a full theory of quantum gravity - a theory that has so far been missing and $P S-B E C$. Here are some possible encountered answers: (1) Information really destroyed in black holes-perhaps that prohibition of quantum mechanics is wrong; (2) Inside a black hole a new region of phasespace (6D) forms a sort of baby universe, in which information is preserved. If so happened, for me that baby universe consists of the fifth state of matter before the quark-gluon plasma of the time scale of order $n s$-ps. Annihilation $\gamma$-rays (511 $\mathrm{keV}$ ) will be coming out instead of visible light that makes black hole's information what Hawking said event horizon; (3) Other solutions involve theoretical objects called white holes-the opposite of black holes, in which the flow of time is reversed and nothing can fall in, only out (information included). Then there is the chance that black holes never quite evaporate - they only shrink down to incredibly small sizes of density more order than $B E C$, thereby preserving the information. Or perhaps information is somehow copied from inside a black hole to outside, so that when the black hole is destroyed the outside copy remains; and finally (4) there are proposals in which information is encoded on a black hole's event horizon in various ways-Hawking's idea falls into this category. He said, “black holes aren’t as black as they are painted. They are not the eternal prisons they were once thought. Things can get out of a black hole both on the outside and possibly come out in another universe”. This is again a puzzle unless physicists understood the reality completely.
On September 2015, LIGO (Laser Interferometer Gravitational-wave Observatory) Scientific collaboration, USA and Virgo collaboration, Italy together observed a transient gravitational wave signal (sound) produced in merging spiral black holes that matches the waveform predicted in general relativity by Einstein almost 100 years ago [22]. These wonderful observations prove the existence of binary stellar-mass black hole systems and the success of my thought of fifth state of matter of primordial universe. (Please note that this paragraph is included after I have read the reference 22 which is published after my article has been accepted).

\section{Acknowledgement}

Author is pleased to acknowledge the financial support from his family and partially from his institute and thankful to Prof. A.P. Mills Jr. for encouraging me in the $P s-B E C$ research.

\section{References}

[1] Callahan, D. 2015. "Hawking: Information Lost in Black Holes Could be Stored in Alternate Universes." STOCKHOLM TECHNOLOGY BLOG. http://www.kth.se/blogs/stockholmtech/2015/08/hawking -information-lost-in-black-holes-could-be-stored-in-altern ate-universes/.

[2] Hawking, S. W., Perry, M. J., and Storinger, A. 2016. "Soft Hair on Black Holes.” Cornell University Library. http://arxiv.org/abs/1601.00921.

[3] Bohr, H., and Nielsen, H. B. 1977. "Hadron Production from a Boiling Quark Soup: Quark Model Predicting Particle Ratios in Hadronic Collisions.” Nuclear Physics B 128 (2): 275.

[4] Bose, S. N. 1924. Zeitschriftfür Physik 26 (1): 178-81.

[5] Adams, C. S., Lee, H. J., Davidson, N., Kasevich, M., and Chu, S. 1995. "Evaporative Cooling in a Crossed Dipole Trap.” Phys. Rev. Lett. 74: 3577. Chu S., Mills A. P. Jr. and Hall J. L. 1984. Phys. Rev. Lett. 52: 1689.

[6] Davidson, N., Lee, H. J., Adams, C. S., Kasevich, M., and Chu, S. 1995. "Long Atomic Coherence Times in an Optical Dipole Trap.” Phys. Rev. Lett. 74: 1311.

[7] Miesner, H. J., Stamper, D. M., Stenger, J., Inouye, S., Chikkatur, A. P., and Ketterle, W. 1999. "Observation of Metastable States in Spinor Bose-Einstein Condensates.” Phys. Rev. Lett. 82: 2228-31. 
[8] Stenger, J., Inouye, S., Andrews, M. R., Miesner, H. J., Stamper, D. M., and Ketterle, W. 1999. "Strongly Enhanced Inelastic Collisions in a Bose-Einstein Condensate Near Feshbach Resonances.” Phys. Rev. Lett. 82: 2422-5.

[9] Inouye, S., Andrews, M. R., Stenger, J., Miesner, H. J., Stamper, D. M., and Ketterle, W. 1998. "Observation of Feshbach Resonances in a Bose-Einstein Condensate.” Nature 392: 151-4.

[10] Ketterle, W., and Miesner, H. J. 1997. "Coherence Properties of Bose-Einstein Condensates and Atom Lasers.” Phys. Rev. A 56: 3291-3.

[11] Chu, S. 1997. "Nobel Lecture: Manipulation of Neutral Particles.” Nobelprize.org, December 8, http://www.nobelprize.org/nobel_prizes/physics/laureates /1997/chu-lecture.html.

[12] Mondal, N. N. 2002. "A Novel Detection System for Laser Cooling of Ortho-Positronium.” Nucl. Inst. \& Meth. Phys. Res. A 495: 161-9.

[13] Kumita, T., Hirose, T., Irako, M., Kadoya, K., Matsumoto, B., Wada, K., Mondal, N. N. et al. 2002. "Study on Laser Cooling of Ortho-Positronium." Nucl. Inst. \& Meth. Phys. Res. B 192: 171-5.

[14] Mondal, N. N., Hamatsu, R., Hirose, T., Irako, M., Kumita, T., Ijima, H. et al. 1999. Appl. Surf. Sci. 149:
269-79.

[15] Hirose, T., Iijima, H., Irako, M., Kajita, M., Kobayashi, K., Kumita, T., Mondal, N. N., Wada, K., and Yabu, H. 2000. In Proceedings of the International Conference on LASERS, 437.

[16] Mills, A. P. Jr. 2010. "Purifying Positronium Could Help Develop Gamma Ray Lasers and Produce Fusion Power.” Science Daily, May 4.

[17] Mills, A. P. Jr. 2002. "Positronium Molecule Formation, Bose-Einstein Condensation and Stimulated Emission." Nucl. Inst. \& Meth. Phys. Res. B 192: 107-16.

[18] Surko, C. M., and Greaves, R. G. 2004. Phys. Plasmas 11: 2333.

[19] Cassidy, D. B., and Mills, A. P. Jr. 2007. "The Production of Molecular Positronium.” Nature 449: 195-7.

[20] Wang, Y. H., Anderson, M. B., and Clark, C. W. 2014. "Spinor Bose-Einstein Condensates of Positronium." Phys. Rev. A 89: 043624.

[21] Avetissian, H. K., Avetissian, A. K., and Mkrtchian, G. F. 2014. "Self-amplified Gamma-ray Laser on Positronium Atoms from a Bose-Einstein Condensate.” Phys. Rev. Lett. 113: 023904.

[22] Abbott, B. P. et al. 2016. "Observation of Gravitational Waves from a Binary Black Hole Merger.” Phys. Rev. Lett. 116: 061102-1-16. 